\title{
PEQUENOS EMPREENDEDORES CRIATIVOS: estratégias de marketing bem-sucedidas nos últimos dez anos

Walney Barbosa ${ }^{1}$

Jane Marques ${ }^{1}$

${ }^{1}$ Universidade de São Paulo 


\section{PEQUENOS EMPREENDEDORES CRIATIVOS: estratégias de marketing bem-sucedidas nos últimos dez anos}

Resumo: Dadas as dificuldades enfrentadas por pequenas e médias empresas das indústrias criativas brasileiras para se estabelecer no mercado nacional, o presente trabalho buscou realizar um levantamento da literatura nos últimos dez anos de estratégias de marketing bem-sucedidas utilizadas por empresas criativas ao redor do mundo que poderiam ser localmente replicadas ou adaptadas. A partir de uma revisão integrativa da literatura, 48 artigos científicos dos mais diferentes periódicos de ciências sociais aplicadas foram selecionados por sua relevância, classificados e avaliados criticamente sob uma ótica qualitativa. O resultado foi a identificação, por parte dos autores, de 3 tipos de ações estratégicas de maior destaque adotadas pelas empresas: 1) Ir além da manifestação física do produto criativo; 2) Explorar o potencial comercial de comunidades online e 3) Usar dados como fonte de estratégias de marketing. Cada tipo de estratégia foi desenvolvida e ilustrada com aplicações práticas dos estudos revistos.

Palavras-chave: criatividade, indústrias criativas, games, estratégia de marketing.

\section{$1 \quad$ Introdução}

Empresas de diversos tipos e tamanhos, em geral, possuem desafios relacionados à elaboração de ofertas que precisam gerar valor para potenciais consumidores, garantindo sua lucratividade a curto prazo e sua sobrevivência a médio e longo prazos. Para tal, estratégias e ações sistematizadas são amplamente aplicadas nos domínios do marketing, em busca de ideais como: lealdade dos consumidores, incremento da qualidade dos produtos, excelência em prestação de serviços, gestão eficiente de canais de comunicação ou distribuição, capacidade de previsão de demanda, entre outros.

No entanto, empresas que trabalham com a produção, desenvolvimento ou distribuição de produtos como jogos de videogame, filmes ou músicas, apresentam um fator incremental de complexidade que muitas vezes chega a limitar ou até mesmo inviabilizar ações padronizadas e tradicionais de solução de problemas de marketing. Este fator incremental é a natureza da principal matéria prima desta indústria: a criatividade humana. Estas empresas são classificadas pelo Departamento de Cultura, Mídia e Esportes do Reino Unido, como empresas das chamadas indústrias criativas (DCMS, 1998). Essa classificação possui respaldo internacional por seu pioneirismo no detalhamento das atividades criativas desse tipo de empresa, especialmente na Inglaterra, na década de 1990.

Por um lado, há a necessidade de controlar, estabelecer prazos e objetivos claros para reduzir riscos e garantir estabilidade (fator 'indústria'). Por outro, as ofertas dessas empresas só podem ter seu valor maximizado por meio de um ambiente livre, enriquecedor e que destaque o intelecto humano como protagonista da transformação (fator 'criatividade') (BENDASSOLLI et al., 2008). As empresas das chamadas indústrias criativas sofrem, por definição, com profundas contradições e desafios. Para compreensão da abrangência das indústrias criativas no Brasil, adota-se a definição da FIRJAN (2019) que agrega treze segmentos em quatro áreas: Consumo, Mídias, Cultura e Tecnologia.

A situação tende a ficar ainda mais difícil para pequenas e médias empresas pelas dificuldades de acesso a recursos (humanos, tecnológicos e/ou financeiros). No Brasil, a 
indústria de jogos digitais, por exemplo, é composta por pequenos e médios desenvolvedores que não estão entre as principais empresas do cenário global, representando somente $1 \%$ de todo o dinheiro movimentado no mundo (NEWZOO, 2019). De modo geral, as indústrias criativas brasileiras (somando publicidade, moda, livros, música, teatro, cinema, games e outras) são responsáveis somente por 2,61\% do PIB nacional (FIRJAN, 2019).

Levando em consideração o advento da internet, mudanças estruturais no fluxo de informações e na forma como produtos da indústria estão sendo consumidos e comercializados, este trabalho procura responder ao seguinte problema de pesquisa:

Quais são as estratégias de marketing que estão impulsionando o mercado global das indústrias criativas e que podem ser replicadas por nossos micro e pequenos empreendedores brasileiros?

Sendo assim, o objetivo central do presente trabalho é: explorar padrões de estratégias de marketing bem-sucedidas nas indústrias criativas nos últimos 10 anos, de modo a contribuir com o campo de conhecimento que visa dar suporte e desenvolver o mercado das indústrias criativas em território nacional, em especial para pequenos e médios empreendedores criativos.

Para cumprimento do objetivo central, este trabalho conta com 3 (três) objetivos secundários: a) selecionar referenciais que tratam do tema em forma de estudos de caso, artigos teórico-empíricos e outros tipos de estudos científicos; b) explorar qualitativamente possíveis padrões de estratégias de marketing, entre os estudos levantados; e c) elencar aspectos comuns, em uma abordagem exploratória e qualitativa de boas práticas que podem servir como referência a pequenos empreendedores dessa indústria no mercado nacional.

\section{$2 \quad$ Referencial Teórico}

A presente fundamentação se concentra em dois grandes conceitos-chave que são entendidos como pré-requisitos para o adequado entendimento das demais seções do trabalho: a) indústrias criativas e b) estratégias de marketing. Demais conceitos estão pontualmente esclarecidos, à medida que se façam necessários.

Para iniciarmos a discussão sobre as indústrias criativas, recupera-se Runco e Jaeger (2012) que investigaram distintas definições de criatividade na literatura acadêmica. Dentre as opções apresentadas por esses autores, a que mais se destaca por sua capacidade de generalização e abrangência é a de Stein (1953, p. 311):

O trabalho criativo é um trabalho novo aceito como sustentável, útil ou satisfatório por um grupo em algum momento no tempo. Por novo quero dizer que o produto criativo não existiu anteriormente com a mesma forma... O trabalho é novo na medida em que se desvia do tradicional ou do status quo. Isto dependerá da natureza do problema a ser atacado, o nível de conhecimento preexistente no campo de conhecimento em questão e as características tanto do indivíduo criador quanto dos indivíduos com os quais o mesmo se relaciona.

Essa definição vai ao encontro do que Bendassolli et al. (2009) identificam como empresas pertencentes a essas indústrias, que geram valor comercial a seus produtos e serviços: 
[Indústrias criativas são] Atividades que têm a sua origem na criatividade, competências e talento individual, com potencial para a criação de trabalho e riqueza por meio da geração e exploração de propriedade intelectual [...]. As Indústrias Criativas têm por base indivíduos com capacidades criativas e artísticas, em aliança com gestores e profissionais da área tecnológica, que fazem produtos vendáveis e cujo valor econômico reside nas suas propriedades culturais (ou intelectuais).

De acordo com Bendassolli et al. (2009), portanto, há quatro características desses negócios criativos, a saber:

- Característica número 1 - Criatividade [prática]: é a expressão da capacidade humana de realizar, manifestada pela criação de produtos tangíveis. Ou seja, é a capacidade que uma pessoa possui de manipular objetos do mundo externo em um processo simultâneo de desenvolvimento de seus recursos pessoais, suas fantasias e seus desejos (WINNICOTT, 1975).

- Característica número 2 - Valorização da arte pela arte: ainda que haja potencial conflito entre as instâncias criativas e as instâncias funcionais (BLYTHE, 2001; BOURDIEU, 1993) típicas dos sistemas de gestão das indústrias tradicionais, as concepções estéticas e artísticas das Indústrias Criativas têm forte influência sobre as escolhas e o direcionamento de recursos (CAVES, 2000).

- Característica número 3 - Uso intensivo de novas tecnologias: a indústria da música é um bom exemplo de como as novas tecnologias revolucionaram a forma como a música tem sido produzida, distribuída e consumida, o que já chegou a levar grandes conglomerados de gravadoras à falência (MOLTENI; ORDANINI, 2003). Outro exemplo é a falência do modelo de distribuição da Blockbuster, que deu espaço a tecnologias mais avançadas como os serviços de streaming de filmes e séries da Netflix (MERIGO, 2011).

- Característica número 4 - Uso extensivo de equipes polivalentes: como a criatividade e a falta de linearidade do processo de produção ditam o ritmo dessas organizações, há uma necessidade natural de que os profissionais envolvidos tenham diferentes habilidades, especialidades e que consigam manejar os mais diferentes recursos, exigindo flexibilidade e domínio de múltiplas disciplinas.

As Indústrias Criativas são, na prática, empresas que lidam, entre outras possibilidades, com atividades relacionadas ao teatro, ao cinema, à música, às artes plásticas e, também, aos videogames (BENDASSOLLI et al., 2009).

O mercado brasileiro, segundo a FIRJAN (2019, p. 4), concentra treze segmentos criativos, agregados em quatro grandes áreas criativas, a saber: "Consumo (Design, Arquitetura, Moda e Publicidade \& Marketing), Mídias (Editorial e Audiovisual), Cultura (Patrimônio e Artes, Música, Artes Cênicas e Expressões Culturais) e Tecnologia (P\&D, Biotecnologia e TIC)".

Em termos de empregabilidade, no Brasil, esses segmentos ainda estão concentrados na Região Sudeste, sendo que 50\% dos profissionais trabalham em São Paulo e Rio de Janeiro; principalmente na área de Tecnologia. Esta área se destaca pelas maiores remunerações e pequeno aumento de contratações (FIRJAN, 2019), considerando que nos últimos dois anos houve queda em termos de admissões de profissionais criativos. A área de Tecnologia, que é a 
que teve maior número de contratações nesse último biênio, acompanha indicadores mundiais, considerando a emergência da economia digital e da indústria 4.0 (FIRJAN, 2019).

O SEBRAE - Serviço Brasileiro de Apoio às Micro e Pequenas Empresas (2019), estima que em torno de $99 \%$ de empresas no Brasil sejam micro e pequenas empresas e, estas por sua vez, respondem por $52 \%$ dos empregos formais no setor privado. Destas empresas, 9,1 milhões de empreendedores são MEI (Microempreendedores individuais). Entre as mais de 400 categorias de atividades profissionais dos MEIs, temos uma série de atividades que se conectam às indústrias criativas como promotores de eventos, músicos, artistas plásticos, artesãos de itens de moda, desenvolvedores de jogos, entre outros (PORTAL DO EMPREENDEDOR, 2019). A falta de conhecimento de mercado, de capital e dificuldades em planejar as próximas etapas do negócio (incluindo estratégias de marketing) são algumas das principais dificuldades que estes empreendedores individuais e pequenas empresas tendem a enfrentar para desenvolver suas atividades (LOPES, BOLDRIN, 2013).

Com esses esclarecimentos acerca das indústrias criativas, partimos para o entendimento de estratégias de marketing, que consistem, por sua vez, no planejamento a ser adotado por uma instituição para divulgar sua marca, seus produtos e/ou serviços, visando manter e atrair novos clientes (FERRELL et al., 2000). Considera-se que a elaboração dessas estratégias deve seguir o planejamento estratégico da empresa, para que haja sinergia e direcionamento correto com a missão, visão e metas estabelecidas (FERRELL et al., 2000; KOTLER; ARMSTRONG, 2007).

Deve-se selecionar o(s) mercado(s)-alvo a ser(em) atendido(s) e, a partir disso, qual o desenvolvimento de composto de marketing, a fim de satisfazer as necessidades desse(s) consumidor(es) (FERRELL et al. 2000). O principal objetivo das estratégias de marketing é, portanto, que a organização atue orientada para os clientes, oferecendo a estes entregas de maior valor para conquistá-los em relação aos concorrentes (CHURCHILL; PETER, 2005; KOTLER; ARMSTRONG, 2007).

Esse planejamento deve ser revisto continuamente para garantir que os resultados esperados para cada ação prevista estão sendo realmente positivos e para ajustar o que precisar de adequação. Varadarajan (2010, p. 128), a partir de um extenso trabalho de revisão, propõe uma definição global para o termo 'estratégia de marketing':

Estratégia de marketing refere-se ao padrão integrado de decisões de uma organização que especifica suas escolhas cruciais em relação aos mercados a serem atendidos e segmentos de mercado a serem atingidos, atividades de marketing a serem realizadas e a maneira de desempenho dessas atividades e a alocação de recursos de marketing entre mercados, segmentos de mercado e atividades de marketing para a criação, comunicação e/ou entrega de um produto que ofereça valor aos clientes nas trocas com a organização e, assim, permita à organização alcançar objetivos específicos.

Esta é, portanto, a definição adotada pelo presente estudo para investigar, selecionar, categorizar, desenvolver e qualificar estratégias de marketing aplicadas nas empresas das indústrias criativas que foram objeto de revisão. 


\section{$3 \quad$ Metodologia}

Para o presente trabalho utilizamos do método de revisão de literatura ou revisão bibliográfica que, segundo Alves-Mazzotti (2002), refere-se à análise de publicações anteriores sobre o tema e/ou temas correlatos e à discussão desse material.

Embora não haja muita sinergia entre os estudos nas ciências sociais, haja vista a fragmentação da produção e a grande variedade de abordagens teóricas e metodológicas adotadas, Alves-Mazzotti (2002) destaca a importância de se investigar estudos já realizados para avaliar a adequação teórica e metodológica adotadas e relativizar as inconsistências dos resultados apresentados.

Tendo em vista o objeto desta pesquisa - as indústrias criativas -, buscamos sintetizar o conhecimento e consideramos estudos científicos que também tivessem aplicações práticas para servir como parâmetros. Mesmo sendo uma revisão exploratória inicial, adotamos alguns procedimentos para evitar imprecisões ou vieses na busca.

Partimos da seguinte pergunta norteadora para esta revisão: Quais são os trabalhos científicos que estão tratando de fenômenos relacionados a estratégias de marketing que podem estar impulsionando o mercado mundial das indústrias criativas nos últimos 10 anos?

A base de dados escolhida foi o Google Scholar, filtrando trabalhos publicados até junho de 2018 (últimos 10 anos), utilizando combinações booleanas dos seguintes termos-chave: marketing strategies, creative industries, game industry, film industry, music industry e case study. A busca retornou uma seleção de 48 artigos, que foram lidos na íntegra para um primeiro contato exploratório sobre o tema, após avaliação de títulos (primeiro critério de corte), resumos (segundo critério de corte) e relevância para responder à pergunta norteadora (terceiro critério de corte). Para estudos futuros há a intenção de ampliar a busca para outras bases de dados e avaliar por completo distintos trabalhos que possam ser incluídos, visando a uma análise sistemática e integrativa.

Dessa forma, coletamos os seguintes dados: ano de publicação (critério de corte, considerando apenas os últimos dez anos), nome do trabalho, resumo, objetivo, método de pesquisa utilizado e resultados obtidos.

Os trabalhos selecionados como relevantes foram lidos e classificados em 5 (cinco) categorias: cadeia de valor e distribuição, papéis e comportamentos do consumidor, cultura empreendedora e de marketing digital, comunidades digitais e ciência de dados aplicada ao marketing. Apresentamos a discussão dos resultados na próxima seção do presente artigo.

\section{$4 \quad$ Análise dos Resultados}

Com base nos procedimentos de revisão adotados, chegou-se a um consenso sobre a existência de três grandes dimensões estratégicas de marketing que têm influenciado direta ou indiretamente a dinâmica e o progresso das indústrias criativas ao redor do mundo nos últimos dez anos e que podem servir de inspiração para pequenos e médios empreendedores criativos, a saber: a) ir além da manifestação física do produto criativo; b) explorar o potencial comercial de comunidades online; e c) usar dados como fonte de estratégias de marketing. As dimensões 
encontradas sintetizam uma avaliação qualitativa dos estudos que identificamos, sem a pretensão de generalizar ou limitar a existência de outros fenômenos adjacentes.

\subsection{Ir além da manifestação física do produto criativo}

Uma pequena banda de indie rock formada em 2006, nos EUA, The Low Anthem, não se limitou a caminhos tradicionais da indústria da música para criar uma base de fãs fiéis (fazer alguns shows, gravar alguns discos ou tocar nas rádios locais). Conforme achados de um estudo de caso feito por Holt (2011), sua sobrevivência não depende somente dos ouvidos, mas dos olhos das pessoas. A banda realiza regularmente shows que são transmitidos online via múltiplas plataformas, sites especializados e outros parceiros. Também alimenta blogs com conteúdos que criam uma atmosfera mais íntima com os passos dados pela banda e, vez ou outra, disponibiliza partes de novos conteúdos exclusivos a serem lançados como videoclipes ou entrevistas. São formas visuais de contato com o público que superam muito a capacidade de abrangência de shows locais e pequenas turnês realizadas por bandas tradicionais em início de carreira.

Com a incapacidade de tradicionais conglomerados da música de se reinventarem e reagirem aos novos paradigmas impostos pela distribuição online de conteúdo (WIKSTRÖM, 2015), novas formas de distribuição passaram a emergir nos últimos anos. Contemporâneo ao Spotify, por exemplo, a plataforma Bandcamp (lançada 2 anos após, em 2008), estabeleceu novas formas de atender à demanda latente de consumo de música online. Maftei e Girogiannis (2016) chegaram à conclusão de que as principais alavancas de sucesso do modelo de negócio estão associadas à capacidade da empresa de ser ao mesmo tempo uma plataforma online de transmissão de música (gratuita, em boa parte), uma loja online (que permite comprar conteúdos físicos e/ou digitais) e uma plataforma de promoção de artistas (que se sentem satisfeitos e comprometidos com o compartilhamento e comercialização de conteúdos autorais por meio da plataforma). Já o Spotify, líder do segmento, também se utiliza destes recursos, potencializando-os em um processo evolutivo contínuo que leva em consideração o feedback de usuários e de músicos para embasamento de estratégias de distribuição e priorização de conteúdos fornecidos pela plataforma (SKOG; WIMELIUS; SANDBERG, 2018).

Perren (2013) ressalta a obsessão que as empresas de produtos midiáticos historicamente possuem com a entrega de valor através de produtos físicos. Com a queda de popularização de CDs, DVDs, jornais e revistas, a autora argumenta que a falta de entendimento de um novo paradigma de distribuição foi fundamental para a posterior queda das empresas que embasavam a entrega de valor a partir destes meios. O fracasso da luta antipirataria (VELASQUEZ; NEWMAN; MILLER, 2012) também pode ser um reflexo dessa obsessão e inflexibilidade, dando espaço para novos modelos de negócio, como os retratados anteriormente e, também, para novas formas de cobrança pelo valor oferecido. Em trabalhos como o de Günzel-Jensen e Holm (2015), por exemplo, temos condições de conhecer mais sobre os modelos fremium, que fogem ao escopo deste estudo.

Examinando o papel desempenhado entre diversas empresas nas indústrias criativas, também se observa o protagonismo de empresas com fortes elementos tecnológicos em seus modelos de negócio que dificultam a clara separação entre parceiros e competidores. Daidj e 
Jung (2015) estudaram o fenômeno da "coopetição" que, segundo Nalebuff e Brandenburger (1995), é um conceito de criação formado pela união dos termos competição e colaboração, e significa trabalhar em parceria com os concorrentes, visando otimizar características distintivas tanto em pesquisa e desenvolvimento, quanto na produção, distribuição, etc. Empresas como a Netflix, por exemplo, ao mesmo tempo que distribuem conteúdos de terceiros, possuem a capacidade de criação de conteúdos próprios com o auxílio de infraestrutura tecnológica que provê informações qualificadas sobre o comportamento e preferências dos usuários de sua plataforma.

Esta área cinzenta entre cooperação e competição também pode ser ilustrada com a união das indústrias de animação e de videogames na França, que passaram a trabalhar em conjunto para oferecer um ecossistema de ofertas inovadoras e que usufruem das especialidades artísticas e de programação de ambas as indústrias (GANDIA, 2013). Este é um reflexo das tradicionais estratégias da Disney de criação de ecossistemas de ofertas de múltiplas indústrias, incluindo o uso de personagens clássicos em jogos de videogame e também a experimentação de estratégias de product placement e novas formas de publicidade em ambientes virtuais (BOLUDA; PARDO; SUEMANOTHAM, 2010).

Obviamente, não se pode resumir o sucesso de uma empresa às suas características intrínsecas e estratégias próprias. As empresas finlandesas Rovio e Supercell, por exemplo, são protagonistas nas indústrias de jogos mobile por conta de massivos investimentos realizados pelas esferas pública e privada (HARMA, 2013). Chen, Chang e Lee (2014) reforçam estes aspectos por meio de um estudo com 293 empreendedores criativos chineses, demonstrando que o sucesso dessas empresas depende de complexas redes de relacionamento divididas em 4 (quatro) categorias: 1) família e 2) comunidade (para obtenção de recursos iniciais); 3) negócios (para compartilhamento de experiência e expertise na área de atuação); e 4) governo (para investimentos de base e incentivos fiscais).

Em resumo, pode-se entender que a entrega do produto criativo em si - e maximização de sua qualidade intrínseca (a melhor música, filme, livro ou jogo de videogame) não é suficiente para maximização da geração de valor.

\subsection{Explorar o potencial comercial de comunidades online}

A indústria dos videogames pode ser vista, entre as indústrias criativas, como uma das que melhor exploraram o valor comercial das comunidades online até o momento. Os Massively Multiplayer Online Role-Playing (MMORPGs) mudaram completamente a forma como este tipo de produto é consumido (ZACKARIASSON; WILSON, 2010).

Estes tipos de jogos, que colocam simultaneamente milhares de pessoas em um único mundo virtual, de modo que jogadores possam interagir (competindo ou cooperando), foram a base de um fenômeno recente e muito expressivo, denominado e-sports (ou "esportes eletrônicos"). Trata-se uma maneira completamente nova de consumir videogames, pois altera o paradigma de entretenimento em casa, característico dos anos 1990, para um paradigma de entretenimento em grupo iniciado pelos Arcades (máquinas de videogames distribuídas em locais públicos) dos anos 1980 (BOROWY; JIN, 2013). 
Em consonância com a dimensão estratégica anterior, há neste ecossistema a atuação de múltiplos agentes que, quando organizados para oferecer uma adequada experiência de consumo, conseguem trazer satisfação e engajamento por parte dos usuários. São estas as empresas de videogames, governos, fornecedores de tecnologia e infraestrutura, empresas de marketing e comunicação, imprensa, entre outras (SEO, 2013). Seo (2013) ainda reforça que as empresas precisam mudar seu foco do produto em si (no caso, o próprio jogo de videogame) para se concentrar mais em ações de design colaborativo e gestão do ecossistema de $e$-sports para maximização do valor para seus múltiplos atores.

Para além da indústria de jogos, empresas que trabalham com eventos musicais também têm experienciado vantagens em gerenciar e incentivar comunidades online. Hudson et al. (2015) estudaram, a partir de um modelo quantitativo de análise de equações estruturais, o papel que comunidades criadas nas redes sociais tinham no sucesso de festivais de música. A conclusão desses autores foi de que as comunidades online possuem não só papel crucial na divulgação do evento, como também criam sentimentos e expectativas positivas nas pessoas que possuem contato com estas comunidades.

Ainda sobre o mercado musical, o K-pop (estilo de música coreano) se tornou ao mesmo tempo um fenômeno cultural e um ambiente fértil para criação de comunidades online de fãs não só do Oriente como do Ocidente, graças à internet $(\mathrm{OH}, 2013)$. Esse fenômeno proporcionou a determinados grupos de empresas amplas oportunidades de negócio, satisfazendo demandas sedentas por produtos e serviços relacionados ao segmento.

\subsection{Usar dados como fonte de estratégias de marketing}

O termo 'ciência de dados' e a profissão 'cientista de dados' são relativamente novos, mas compreendem um conjunto de competências historicamente estabelecidas. Waller e Fawcett (2013, p. 78) definem ciência de dados como a "aplicação de métodos quantitativos ou qualitativos para resolução de problemas e predição de resultados".

Em artigo publicado, Davenport e Patil (2012) argumentam que esse tipo de cientista é um profissional que possui um conjunto de habilidades técnico-científicas direcionado à resolução de problemas do mundo das organizações.

Uma das pioneiras na utilização de ciência de dados para tomadas de decisões em marketing nas indústrias criativas é a Netflix, que se tornou nos últimos tempos uma referência mundial na criação de filmes e séries com base nos padrões de comportamento de seus consumidores em sua plataforma de vídeos online. Foram as bilhões de interações diárias que permitiram que a Netflix entendesse gostos e preferências sobre certos tipos de narrativas, personagens e temas, dando origem a House of Cards, série norte-americana de propriedade da Netflix, que foi a primeira série da internet a concorrer a nove Emmys (BANDTEC, 2015), considerado o principal prêmio atribuído a programas e profissionais de televisão.

Outra aplicação prática da ciência de dados por parte da Netflix está em seus algoritmos de recomendação de títulos, que se apropriam de uma massiva quantidade de informações para fornecer filmes e séries de acordo com o histórico de cada usuário (AMATRIAIN, BASILICO, 2015). Fernández-Manzano, Neira e Clares-Gavilán (2016) de forma mais taxativa afirmam: “a 
manipulação de grandes bases de dados é a principal estratégia nas indústrias midiáticas e ao mesmo tempo seu maior desafio para expansão e sobrevivência”.

\section{$5 \quad$ Conclusões}

Este estudo teve por objetivo apresentar uma abordagem exploratória de estratégias de marketing utilizadas globalmente entre as indústrias criativas nos últimos dez anos. Neste primeiro levantamento foi possível notar a presença de novos tipos de estratégia de marketing que usam como base a ampla gama de recursos propiciada pelo ambiente digital e tecnológico.

Novas formas de abordar os clientes, explorar o valor das comunidades online e utilizar dados para tomadas de decisão foram alguns bons exemplos que empresas de diversos portes ao redor do mundo trazem para o desenvolvimento das indústrias criativas, possibilitando estender suas aplicações em território nacional.

Os 3 tipos de estratégias identificados neste estudo podem servir como referência a pequenos empreendedores dessa indústria no mercado nacional. Ainda assim, são classificações muito abrangentes e que exigem um considerável esforço de abstração para sua aplicação prática, possivelmente por se tratar de uma primeira tentativa de abordagem exploratória e qualitativa de boas práticas. Observamos, no entanto, a partir dos estudos que referenciamos, que não é possível se estabelecer no mercado das indústrias criativas, independente do porte da organização, sem que estratégias mercadológicas sejam pensadas e praticadas.

Dentre as principais limitações deste trabalho inclui-se a ainda pequena quantidade de estudos que foram avaliados na íntegra, assim como a necessidade de avançar nas buscas em outras bases de dados e ampliar as possibilidades de combinações de termos-chave utilizados.

Outra limitação que pode ser abordada em estudos futuros é a incapacidade de realização de análises de impacto e de significância de cada tipo de estratégia no sucesso das empresas das indústrias criativas, dado o caráter qualitativo e exploratório proposto.

Por fim, um tratamento de revisão mais abrangente pode trazer à tona novas classificações de estratégias de marketing ou até mesmo implicar na reclassificação das estratégias apresentadas neste artigo, ampliando o leque de possibilidades a serem conhecidos por pequenos e médios empreendedores criativos.

\section{$6 \quad$ Referências}

ALVES-MAZZOTTI, A. J. A "revisão bibliográfica" em teses e dissertações: meus tipos inesquecíveis - o retorno. In: BIANCHETTI, L.; MACHADO, A. M. N. (Org.). A bússola do escrever: desafios e estratégias na orientação de teses e dissertações. São Paulo: Cortez, p. 2544, 2002.

AMATRIAIN, Xavier; BASILICO, Justin. Recommender Systems in Industry: A Netflix Case Study. In: RICCI, Francesco; ROKACH, Lior; SHAPIRA Bracha. (Eds.). Recommender Systems Handbook. Boston, MA: Springer US, p. 385-419, 2015. 
BANDTEC. O que o Sucesso de House Of Cards Deve ao Big Data? Disponível em: http://tecnologia.bandtec.com.br/o-que-o-sucesso-de-house-of-cards-deve-ao-big-data. Acesso em: 09 nov. 2017.

BENDASSOLLI, Pedro F. et al. Indústrias Criativas: definição, limites e possibilidades. RAE - Revista de Administração de Empresas, v. 49, n. 1, p. 10-18, jan.-mar. 2009. Disponível em: http://www.scielo.br/pdf/rae/v49n1/v49n1a03.pdf. Acesso em: 1 jul. 2019.

BLYTHE, M. The work of art in the age of digital reproduction: the significance of the creative industries. JADE, v. 20, n. 2, p. 144-150, 2001.

BOLUDA, Inés; PARDO, Elena; SUEMANOTHAM, Torpong. Product placement in video games as a marketing strategy: an attempt to analysis in Disney company. In: OKAZAKI, S. (Ed.). Advances in Advertising Research, v. 2, Valencia: Instituto Valenciano de Investigaciones Económica, n. 4, 2010. Disponível em: https://dialnet.unirioja.es/servlet/articulo?codigo=3224690. Acesso em: 11 jul. 2019.

BOROWY, Michael; JIN, Dal Y. Pioneering eSport: The Experience Economy and the Marketing of Early 1980s Arcade Gaming Contests. International Journal of Communication, v. 7, p. 2254-2274, 2013.

BOTELHO, Louise L. R.; CUNHA, Cristiano J. C. A.; MACEDO, Marcelo. O método da revisão integrativa nos estudos organizacionais. Revista Eletrônica Gestão e Sociedade, Belo Horizonte, v. 5, n. 11, p. 121-136, maio/ago. 2011.

BOURDIEU, P. The Field of Cultural Production. New York: Columbia University Press, 1993.

CAVES, R. Creative Industries. Harvard: Harvard University Press, 2000.

CHEN, Ming; CHANG, Yu-Yu; LEE, Chia. Creative entrepreneurs' guanxi networks and success: Information and resource. Journal of Business Research, v. 68, p. 900-905, 2015.

CHURCHILL Jr., Gilbert A.; PETER, E J. Paul. Marketing: Criando Valor para os Clientes. 2. ed. São Paulo: Saraiva, 2005.

DAIDJ, Nabyla; JUNG, Jaemin. Strategies in the Media Industry: Towards the Development of Co-opetition Practices? Journal of Media Business Studies, v. 8, p. 37-57, 2015.

DAVENPORT, Thomas H; PATIL, D. J. Data Scientist: The Sexiest Job of the 21st Century. Harvard Business Review, v. 90, n. 10, p. 70-76, Oct. 2012. Disponível em: https://hbr.org/2012/10/data-scientist-the-sexiest-job-of-the-21st-century. Acesso em: 17 nov. 2018. 
DCMS - Department for Digital, Culture, Media and Sport. Creative Industries Mapping Document.

http://www.culture.gov.uk/global/publications/archive_1998/Creative_Industries_Mapping_D ocument_1998.htm. Acesso em: 01 jul. 2019.

FERNÁNDEZ-MANZANO, Eva-Patricia; NEIRA, Elena; CLARES-GAVILÁN, Judith. Data management in the audiovisual industry: Netflix as a case study. El Profesional de la Información, v. 25, n. 4, p. 568-576, jul.-ago. 2016. Disponível em: http://www.elprofesionaldelainformacion.com/contenidos/2016/jul/06.html. Acesso em: 11 jul. 2019.

FERRELL, O. C. et al. Estratégia de Marketing. São Paulo: Atlas, 2000.

FERRELL, O. C.; HARTLINE, Michael D. Estratégia de Marketing. São Paulo: Pioneira Thomson Learning, 2005.

FIRJAN - Federação das Indústrias do Estado do Rio de Janeiro. Mapeamento da Indústria Criativa no Brasil. Rio de Janeiro, fev. 2019. Disponível em: https://www.firjan.com.br/EconomiaCriativa/downloads/MapeamentoIndustriaCriativa.pdf. Acesso em: 01 jul. 2019.

GANDIA, Romain. The Digital Revolution and Convergence in the Videogame and Animation Industries: Effects on the Strategic Organization of the Innovation Process. International Journal of Arts Management, v. 15, n. 2, p. 32-44, 2013.

GÜNZEL-JENSEN, Franziska; HOLM, Anna B. Freemium Business Models as the Foundation for Growing an E-business Venture: A Multiple Case Study of Industry Leaders. Journal of Entrepreneurship, Management and Innovation, v. 11, n. 1, p. 77-102, 2015.

HARMA, Aleksanteri. The Success Factors of the Finnish Mobile Game Industry: a strategic overview - cases Rovio \& Supercell. Trabalho de Conclusão de Curso (Monografia em International Business) - Turku University of Applied Sciences, 2013.

HOLT, Fabian. Is music becoming more visual? Online video content in the music industry. Visual Studies, v. 26, n. 1, p. 50-61, 2011.

HUDSON, Simon et al. The effects of social media on emotions, brand relationship quality, and word of mouth: An empirical study of music festival attendees. Tourism Management, v. 47, p. 68-76, 2015.

KOTLER, Philip; ARMSTRONG, Gary. Princípios de Marketing. 12. ed. São Paulo: Prentice-Hall, 2007. 
LOPES, Josy; BOLDRIN, Vitor. O microempreendedor individual e as dificuldades encontradas na constituição e condução de seus negócios. Um estudo na cidade de Jales-SP. Disponível em http://conic-semesp.org.br/anais/files/2013/trabalho-1000014924.pdf. Acesso em: 25 out. 2019.

MAFTEI, Vlad A.; GEROGIANNIS, Vassilis C. Critical success factors of online music streaming services - a case study of applying the fuzzy cognitive maps method. International Journal of Technology Marketing, v. 11, n. 3, p. 276-300, 2016.

MERIGO, Carlos. Como a Netflix Destruiu a Blockbuster. B9 - Brainstorm 9, 17 fev. 2011. Disponível em: http://www.b9.com.br/20220/como-a-netflix-destruiu-a-blockbusterinfografico/. Acesso em: 01 jul. 2019.

MOLTENI, L.; ORDANINI, A. Consumption patterns, digital technology and music downloading. Long Range Planning, v. 36, n. 4, p. 389-406, 2003.

NALEBUFF, Barry J.; BRANDENBURGER, Adam M. Co-opetition: competitive and cooperative business strategies for the digital economy. Strategy \& Leadership, v. 25, n. 6, p. 28-33, 1997.

NEWZOO. Brazil Games Market 2018. Disponível em: https://newzoo.com/insights/infographics/brazil-games-market-2018/. Acesso em: 15 jul. 2019.

$\mathrm{OH}$, Inglyu. The Globalization of K-pop: Korea's Place in the Global Music Industry. Korea Observer, v. 44, n. 3, Autumn, p. 389-409, 2013. Disponível em https://www.researchgate.net/publication/296774877. Acesso em: 11 jul. 2019.

PERREN, Alisa. Rethinking Distribution for the Future of Media Industry Studies. Cinema Journal, v. 52, n. 3, p. 165-171, 2013.

PORTAL DO EMPREENDEDOR. Estatísticas. Disponível em http://www.portaldoempreendedor.gov.br/estatisticas. Acesso em: 25 out. 2019.

RUNCO, Mark A.; JAEGER, Garrett J. The Standard Definition of Creativity. Creativity Research Journal, v. 24, n. 1, p. 92-96, 2012.

SEBRAE - Serviço Brasileiro de Apoio às Micro e Pequenas Empresas. Pequenos negócios em números. Disponível em http://www.sebrae.com.br/sites/PortalSebrae/ufs/sp/sebraeaz/pequenos-negocios-emnumeros, 12e8794363447510VgnVCM1000004c00210aRCRD. Acesso em: 25 out. 2019.

SEO, Yuri. Electronic sports: A new marketing landscape of the experience economy. Journal of Marketing Management, v. 29, n. 13-14, p. 1542-1560, 2013. 
SKOG, Daniel.; WIMELIUS, Henrik.; SANDBERG, Johan. Digital Service Platform Evolution: How Spotify Leveraged Boundary Resources to Become a Global Leader in Music Streaming. In: HAWAII INTERNATIONAL CONFERENCE ON SYSTEM SCIENCES, 51. 2018, Big Island, Hawaii. Proceedings... Waikoloa: IEEE, p. 4564-4573, 2018.

SOUZA, Marcela T.; SILVA, Michelly D.; CARVALHO, Rachel. Revisão integrativa: o que é e como fazer. Einstein, São Paulo, v. 8, n. 1, p. 102-106, 2010. Disponível em: http://www.scielo.br/pdf/eins/v8n1/pt_1679-4508-eins-8-1-0102. Acesso em 01 jul. 2019.

STEIN, Morris I. Creativity and culture. Journal of Psychology, v. 36, n. 2, p. 311-322, 1953.

VARADARAJAN, Rajan. Strategic marketing and marketing strategy: domain, definition, fundamental issues and foundational premises. Journal of the Academy of Marketing Science, v. 38, n.2, p. 119-140, Apr. 2010.

VELASQUEZ, Nicole F.; NEWMAN, Hannah; MILLER, Scott. The Impact of a Secondary Market On Video Game Purchase Intentions. Review of Business Information Systems, v. 16, n. 3, p. 103-112, Third Quarter 2012.

WALlER, M. A.; FAWCETT, S. E. Data Science, Predictive Analytics, and Big Data: a revolution that will transform supply chain design and management. Journal of Business Logistics, v. 34, n. 2, p. 77-84, 2013.

WIKSTRÖM, Patrik. The Adaptive Behavior of Music Firms: a music industry feedback model. Journal of Medial Business Studies, v. 6, n. 2, p. 67-96, 2015.

WINNICOTT, D. W. O Brincar e a Realidade. Porto Alegre: Artmed, 1975.

ZACKARIASSON, Peter; WILSON, Timothy. Paradigm shifts in the video game industry. Competitiveness Review, v. 20, n. 2, p. 139-151, 2010. 